\title{
Solar Wind and Its Interaction With the Magnetosphere
}

\author{
Charles P. Sonett
}

\author{
Space Sciences Division NASA, Ames Research Center, Moffett Field, Calif.
}

\author{
(Received February 3, 1965)
}

\begin{abstract}
A short summary of the properties of the solar wind, as they apply to the interaction process with the earth's magnetosphere, is given. Necessary conditions on the bow shock are discussed and are compared to experimental evidence from several space probes. The importance of the downstream or post-shock flow is emphasized. Power spectra and details of the turbulence in this region are reviewed. Limitations due to the very preliminary status of IMP data analysis are emphasized.
\end{abstract}

\section{Introduction}

This paper summarizes some of the properties of the solar wind as they are known from space probe data and attendant physical processes as they may be regarded in light of our generally rudimentary knowledge of collision-free plasmas. A pervasive aspect of the solar wind is its interaction with the magnetosphere of the earth and the subsequent generation of geophysical processes which are noted on the surface of our planet.

It is understood that the solar wind flows nearly continuously and that the outward flow carries along the solar magnetic field. The rotation of the sun results in the field's being drawn out in a spiral pattern whose angle depends upon the velocity of the wind. Although the primary source of energy for the interaction of the solar wind with the magnetosphere comes from the convective energy of the wind, a small additional increment may be found from the rotation of the earth through a drag interaction, such as that proposed by Axford and Hines [1961].

The simplest view of the total phenomenon is that the magnetosphere presents an obstacle to the passage of the solar wind in the manner of a blunt body in aerodynamic flow. The interruption of flow results in a shock wave, followed by a shocked gas region and boundary layer of subsonic flow around the magnetosphere, with the free surface of the magnetosphere itself determining the detailed flow properties near the boundary.

The validity of this aerodynamic point of view is based primarily upon two factors. First, though entirely collision-free over a scale determined by the magnetosphere, the solar wind carries along a magnetic field which is thought to organize the gas into collective

${ }^{1}$ Paper was presented at the ULF Symposium, Boulder, Colo. 17-20 August 1964. motion, i.e., to propagate linear and angular momenta from particle to particle. Second, the field appears to have an energy density comparable to the thermal energy of the solar wind gas, but entirely ignorable compared to the energy of bulk convective flow. Thus, the flow against the magnetosphere is often regarded as a high Mach number instance of aerodymanic flow with the electrodynamic conditions being subsidiary. Though the interaction is magnetohydrodynamic, the flow properties are taken in this view to be essentially aerodynamic.

\section{Solar Wind}

Mariner data are the most complete available in regard to the properties of the solar wind. From these data typical gas parameters indicate a volume density of $\sim 10$ per $\mathrm{cm}^{3}$, a temperature of $\sim 10^{5}{ }^{\circ} \mathrm{K}$, a magnetic fleld of 5 gamma, and a streaming velocity of $400 \mathrm{~km} / \mathrm{sec}$ as being typical of the wind, though the actual value of these parameters fluctuates markedly from time to time. For example, in the experiment of Snyder and Neugebauer [1964] the velocity ranged from 300 to $800 \mathrm{~km} / \mathrm{sec}$ for 90 percent of the data, the temperature reached extremes of several hundred thousand degrees Kelvin, while the magnetic field attained values as high as 20 gamma.

From these measurements we calculate a mean-free path ranging from 1 to $10 \mathrm{AU}$ depending upon the temperature and density values. The electrical conductivity is some $6 \times 10^{14} \mathrm{esu}, 10^{-3}$ that of copper, nevertheless, an extreme value over the cosmic dimensions characteristic of interplanetary space. The absolute viscosity of the solar wind gas is some $10^{-3}$ $\mathrm{g} / \mathrm{cm}$-sec. The kinematic viscosity is about $10^{20}$ $\mathrm{cm}^{2} / \mathrm{sec}$, which is to be compared to air at standard temperature and pressure for which the value of kinematic viscosity is $1.5 \times 10^{-1} \mathrm{~cm}^{2} / \mathrm{sec}$. Since the kine- 
matic viscosity measures the ratio of viscous to inertial forces, it is seen that the viscosity is extremely high and that the solar wind is indeed a very sticky gas, even though the absolute viscosity is low. Another way to regard the kinematic viscosity is to understand it in terms of the transport of momenta, i.e., the diffusion of momentum across a region, this having an extremely large rate because of the long mean-free path. Thus, one is faced with a somewhat paradoxical situation that the more rarefied the gas the higher the kinematic viscosity and the greater the "stickiness." The aerodynamic Reynolds number which is given by the velocity times a characteristic length divided by the kinematic viscosity also measures the effective viscosity of the gas. For the solar wind, the Reynolds number, $R_{n}$, is of the order of $10^{3}$ over a scale size of the magnetosphere. For smaller scale sizes associated with detailed phenomena in the interaction region, the Reynolds number is reduced accordingly. For magnetospheric scale size, $R_{n}$ approaches the critical number for the onset of ordinary turbulent flow. It is unfortunately not possible to extrapolate exactly from this because the magnetic field contained in the solar wind will inhibit the onset of turbulence, kinematic viscosity being reduced by the decrease transport across the field lines. It is possible that the inhibition of transport so decreases the viscosity that at all times the flow of the solar wind against the magnetosphere is highly fluid.

Transverse velocity spectra can only be inferred from Mariner data. These are usually taken to be the same as the longitudinal, isotropy of the velocity distribution being assumed. (The Mariner probe was pointed at the sun and could not measure transverse velocity directly.) It is also indicated from Mariner that some $\alpha$ particles are present in the solar wind. This conclusion is supported by Explorer 14 plasma data [Wolfe and Silva, 1964] taken during the flight of Mariner II on October 7, 1962. Solar wind electrons have never been detected in deep space, but would be expected to be transported with the same bulk velocity as that of the ions, otherwise large currents which are not observed would exist in interplanetary space. Actually, the velocity distribution for electrons in thermal equilibrium with the ions has a mean value lying between 1.3 and $3.7 \times 10^{8} \mathrm{~cm} / \mathrm{sec}$, based upon the ion temperatures of the Mariner and a ratio of specific heats of 2 . Therefore, the bulk and thermal velocities become comingled and their roles tend to be reversed from the ion case. Because of their higher mobility, electrons should be important in transport processes, such as heat conduction and kinematic viscosity.

Properties of the solar wind vary strongly from the base of the corona where it is created out to 1 astronomical unit. At the orbit of earth the Debye length, using the Mariner ion temperature of $10^{5}{ }^{\circ} \mathrm{K}$ and a volume density of $10 / \mathrm{cm}^{3}$, is about $7 \mathrm{~m}$. Although such a distance is large compared to the dimensions of present day spacecraft, this parameter must be referred to the streaming velocity of the wind. Thus, random fluctuations taking place within a Debye sphere occur in times of the order of microseconds and are unobservable, lying outside of the passband of present day magnetometers and plasma probes. Mean collision times are perhaps a day for protons. The greater mobility of electrons means that the collision times are more likely on the order of minutes to an hour.

The flow of the solar wind is often taken to be sheetlike in the plane of the ecliptic, though there is no known spacecraft test of this property. Some evidence for a dropoff in solar wind bulk flow with ecliptic latitude comes from comet tails observed out of the plane of the ecliptic, indicating a dropoff in solar wind forces [Beyer, 1953]. The magnetic field entrained in the solar wind is swept into a spiral pattern according to arguments first given by Parker [1958] which involves the solar rotation. For a nonrotating sun, the field lines would be expected to be radially outward from the sun.

The existence of magnetic fields in interplanetary space was suspected long before spacecraft flight. In particular, the 11-year modulation of the galactic component of cosmic rays, Forbush decreases, and 27-day and diurnal variations indicated the existence of a magnetic modulating mechanism sometime before the first spacecraft experiments on Pioneer V. Variations in the intensity and direction of the interplanetary field are now known from the Mariner measurement [Davis, Smith, Coleman, and Sonett, 1964] to show a marked 27-day effect (fig. 1), which reflects a view of the radially outward component of the field; variations of 10 gamma over a 27-day period are seen in this figure. The lower part of the figure displays the standard deviation taken over different sampling intervals up to 1 day. A small-scale variability is seen in the field, indicating fluctuations in direction. In figure 2 is shown the interplanetary field as determined by Mariner II in ecliptic spherical coordinates with $\beta$ being ecliptic latitude, $\Lambda$ ecliptic longitude, and the magnitude of the field shown in the lower part of the figure. Here the magnitude displays a 27-day variation, the longitude is plotted against the ideal

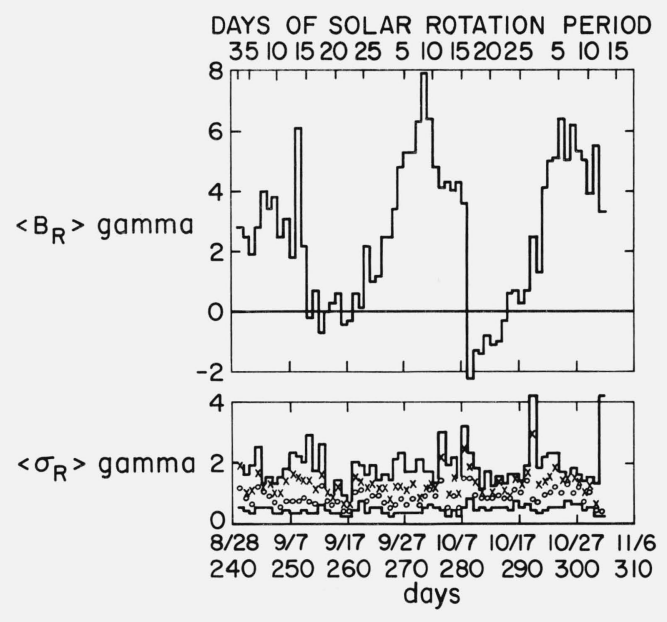

FiguRE 1. One-day average values of the radial component of the interplanetary magnetic field.

A 27-day periodicity is clearly evident in this figure and is presumed to be due to asym. metries in the field resulting from regions of high field on the sun. In the lower part of metries in the field resulting from regions of high field on the sun. In the lower part of
the figure is shown the standard deviation of the radial component for different average times [Davis, Smith, Coleman, and Sonett, 1964]. 


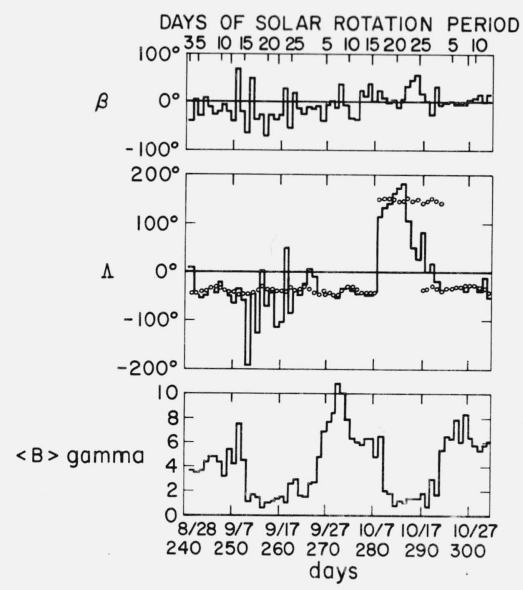

FIGURE 2. Intensity and direction of the interplanetary magnetic field shown in solar ecliptic coordinates; $\beta$ is the ecliptic latitude, $\Lambda$ the longitude measured from the earth-sun line, and $\mathrm{B}$ the intensity of the field.

The 27-day variation is seen to be associated with the magnitude of the field; the longitude is compared to the ideal spiral angle defined by the mean plasma velocity, and, for these 1-day averages, displays one gross change in polarity that lasts a number of days; the latitude which measures the out-of-the-ecliptic component shows continuous variations even over the average times used here [Davis, Smith, Coleman, and Sonett, 1964].

angle of the magnetic field as determined by the solar wind bulk velocity, and the ecliptic latitude shows large variations in and out of the ecliptic component of the field. The general character of these data indicates that over large scales the field takes on the theoretical spiral angle direction and that over periods of a day or less gross variability in direction is to be seen. Lastly, it is primarily the magnitude of the field which displays the 27-day variation rather than direction as indicated in the lower part of figure 2. Fluctuations of the type displayed in the Mariner data indicate that the magnetic field is in approximate energy equilibrium with the thermal energy of the solar wind gas, so that in a frame of reference moving with the solar wind, the field and gas display equipartition, the thermal motions of the gas being shared with a disorder of the magnetic field.

That the interplanetary magnetic field is closely associated with events which take place in the atmosphere of the sun has been suggested by Greenstadt [1963] who has shown a close correlation between both the coronal green line integrated index and plage index and the intensity of a model field based upon Pioneer V. An unexplained aspect of these data is a time dependence indicating a 5- to 7-day delay between an increase in solar indices and the corresponding field increase at the spacecraft.

\section{Magnetospheric Boundary}

Prior to the introduction of the shock concept of stopping the solar wind by the magnetosphere, most investigations of the termination consisted in solving the problem of free-molecule flow of a solar wind, without a magnetic field, against a plasma-free magnetospheric cavity. A number of investigators have made reasonably exact calculations of the shape of the cavity formed by the pressure of this free molecule flow, and the results of their various techniques are not grossly different. All results display neutral points corresponding to where the polar line of force is matched to the surface field of the boundary. Further, all solutions display the characteristic doubling of the magnetic field due to the termination and the consequent flow of surface currents. Since the model used for these calculations has no field carried in the solar wind, the matching of magnetic boundary conditions is simple except for the surface being a free boundary. Clearly, the surface field must be wholly contained in the boundary and the normal component is zero. Seasonal effects cause the surface to change slightly as pointed out by Spreiter and Summers [1963]. They calculated the boundary shape for the case of a wind flowing obliquely, corresponding to seasonal changes. Such changes, they found, cause small variations in the position of the polar neutral points and in the position of the stag. nation point. However, they found the tail of the magnetosphere to lie in the plane of the ecliptic regardless of seasonal change. Such changes cause the position of the polar neutral points and of the stagnation point to change slightly.

Calculations of the type discussed here are important since a precise boundary appears often even though a much more complicated overall view evolves from current experiments. Indeed even the early results of Pioneer I were not in agreement with the view of a simple smooth Chapman-Ferraro boundary.

\section{Bow Wave}

In collision dominated flow, a shock wave will form when the local speed of flow exceeds the sound velocity. Such a wave is a manifestation of the role of the fluid motion in transmitting changes in the fluid rather than by means of sound waves. In this primitive view there is no essential difference between shock waves in free motion and those that occur before an obstacle which is placed within the fluid, except that downstream of the bow wave a region of subsonic flow of great complexity is developed which may expand back to the free-stream supersonic conditions far from the obstacle. This cannot take place, for example, in the one-dimensional shock tube.

The bow wave is greatly complicated by several factors which do not exist in the ordinary collision dominated gas dynamic shock wave. The two issues complicating the physics of this problem are the magnetohydrodynamics and the collisionless property of the gas. Introducing these problems also suggests that shock stability and the modes of propagation of the fluid need to be considered. The energetics of the shock wave are determined primarily by the flow energy of the wind since the thermal and magnetic energies of the gas are so much lower. The bulk flow energy is some $10^{-9}$ to $10^{-8} \mathrm{ergs} / \mathrm{cm}^{3}$ which is usually one to two orders greater than that of either the thermal or magnetic energies. Because of this, it is plausible to expect some similarity to aerodynamic flow in the 
gross sense. Spreiter and Jones [1963] have calculated a model shock based on an aerodynamic theory for a blunt body as developed earlier. Using values for the ratio of specific heats of $5 / 3$ and 2 and restricting their considerations to regions near to the stagnation point, they arrive at shock surfaces which are similar to those in the true aerodynamic case.

Modifying the Spreiter-Jones calculation to the MHD case requires that the interplanetary field be included. Walters [1964] studied the combination of a weak interplanetary field together with the rapid changes in direction. He developed the downstream parameters of the shock from assumptions regarding the upstream or solar wind properties. As expected he found that the downstream properties varied with the free-stream parameters. In his calculation he used the de-HoffmanTeller [1950] equations together with Spreiter and Jones' gross geometry for the aerodynamic shock. The potential weakness of his calculation lies in the lack of self-consistency since, in the computation, the shock surface position is fixed. In reality the consequences of changes in upstream parameters may be reflected in variations in the position of the, shock as well as in the downstream direction of flow of the gas and the direction of the magnetic field.

When the bow wave is considered, it is essential also to take into account the modes of propagation in the solar wind. If an interplanetary field of 10 gamma and a particle density of $10 / \mathrm{cm}^{3}$ are assumed, the Alfvén velocity is about $10^{7} \mathrm{~cm} / \mathrm{sec}$. For a ratio of specific heats of $5 / 3$ and a temperature of $10^{5}{ }^{\circ} \mathrm{K}$ the sound velocity becomes $6 \times 10^{6} \mathrm{~cm} / \mathrm{sec}$. Thus for an ideal field spiral angle of $45^{\circ}$, the slow mode velocity in the direction of flow of the solar wind becomes $1.8 \times 10^{6} \mathrm{~cm} / \mathrm{sec}$ and the fast mode, $1.2 \times 10^{7} \mathrm{~cm} / \mathrm{sec}$. For a solar wind velocity of $400 \mathrm{~km} / \mathrm{sec}$ this means that even for the fast mode the velocity is supersonic, though cases may arise where the sound velocity grows to where the wind is not supersonic to the magnetosphere. Little is known of the detailed properties of the shocks corresponding to these modes, especially regarding their stability. The slow shock is expected to display a decrease in field through the discontinuity, while the Alfvén shock is incompressible and, in effect, a large amplitude linear wave. It seems proper until the stability problems are further clarified to consider that the bow wave is indeed the fast mode shock. Support for this view comes from the calculation of the Mariner October 1962 interplanetary event discussed elsewhere in this paper where the Mach numbers for the flow fit only a fast magnetosonic mode.

An especially important peculiarity of the blunt-body shock is the variation of Mach number over the face of the shock due to the curved geometry. Thus the shock is strongest at the stagnation point where the flow is normal to the surface, and the strength of the shock diminishes on the flanks as the normal component of the flow decreases. The same kind of argument is expected to apply to the blunt body wave with an important added effect due to the presence of the magnetic field. It is easily seen that the presence of the field radically alters the geometry of the shock and makes the problem asymmetric. For example, if we assume the ideal spiral angle of $45^{\circ}$ with respect to the earth-sun line for the direction of the interplanetary field, then the fast mode velocity at the face of the shock will vary in a complicated manner. Depending upon the geometry of the surface of the shock, conditions can be anticipated where the normal component of the flow may be along the direction of the field while on the other side of the magnetosphere the normal component of the flow may turn out to be normal to the local field direction. Thus, the sonic speed may vary over wide ranges on the face of the shock and, indeed, where the flow is along the field, one must consider that the details of the shock and the specification of the local Mach number become difficult to define, in view of the collisionless aspect of the flow. The fact that it is not difficult to find a fast mode velocity for typical solar wind parameters means that from time to time the flow may actually become subsonic for this mode. If the diffusion of hot electrons produced in the shocked flow takes place in the upstream direction, this alone could contribute to the modified sonic velocity's becoming greater than the flow speed of the solar wind.

In attempting to obtain an understanding of collisionfree shocks we have studied an especially favorable shock event which took place in interplanetary space on October 7, 1962. The preliminary results of this calculation have been reported elsewhere [Sonett, Colburn, Davis, Smith, and Coleman, 1964] and here we provide only a summary of the findings. The event noted was a sudden commencement storm with a degenerate main phase and which was seen at the Mariner spacecraft $4.7 \mathrm{hr}$ earlier than the geomagnetic effects. The spacecraft events allow the application of the Rankine-Hugoniot equations and the discontinuity in plasma velocity and magnetic field is treated as a shock wave in a wall-less environment. The change in the field is shown in figure 3 which marks the interplanetary onset. The shock plane appears to be slightly tipped with respect to the earthsun line, denoting an oblique shock. This together with the solar wind aberration required that the most general form of the shock equations be applied. Temperature effects were important in correcting the den-

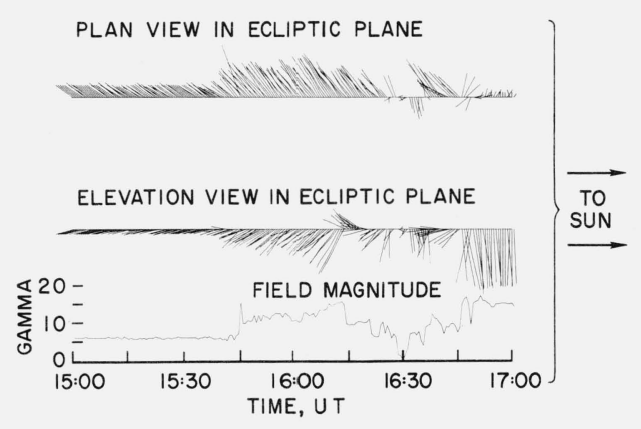

Figure 3. Plan and elevation view of the magnetic field vector for the 2-hour period encompassing the onset of storm conditions.

The spike in the field signifies the onset. The plan view is looking down on the ecliptic, and the elevation view is a cross-section along the earth-sun line. Complementary changes took place in the plasma data at this time. Note the increasing disorder in the field follow. ing the onset of the storm. 
sity. The results of this calculation are to be compared with the measurements which were sufficiently redundant to afford a detailed comparison. Both the measured and computed parameters are given in table 1 which demonstrates an interesting parallel between the different sets of numbers. We believe that within the constraints and limitations of the data available, the results are in reasonable agreement with the assumption of a shock model. Thus the applicability of the shock jump equations is suggested for the collision-free domain, provided the downstream parameters are appropriately averaged before being entered into the computation.

Electrons have not been seriously considered either in the free-stream solar wind or in the shock flow at the magnetosphere. Yet it is expected that their mean thermal velocity, for the same temperature as the ions, would result in fast diffusion. If, in fact, the thermal conductivity is governed by the diffusion of electrons, it may follow that this may limit the thickness of the shocked region in a manner analogous to gas dynamic shocks where both thermal conductivity and viscosity can play a critical role in determining the minimum thickness of the shocked layer.

In MHD shock theory, cases are cited [Sears, 1960] where the formation of a Mach cone is either inhibited, made asymmetrical, or made to appear pointing into the upstream flow. In order to visualize how this can take place reference is made to the group propagation in the anisotropic plasma of the solar wind. Constructing a Friedrichs diagram for the three modes present in a warm plasma allows the group propagation vectors for a solitary pulse to be constructed. Figure 4 is the familiar plot of phase velocities in the case of the magnetic plasma. Figure 5 shows the construction of the envelope for a pulse propagating along the axis defined by the magnetic field. The diagram shows the surface of constant phase for each of the three modes. The fast mode propagates in a slightly asymmetric manner with cylindrical symmetry about an axis defined by the magnetic field. Thus the diagram can be construed as a body of revolution. The propagation velocity across the field is $\left(A^{2}+C^{2}\right)^{1 / 2}$ where $C$ is the sonic velocity and $A$, the Alfvén velocity. The speed in the direction of the magnetic axis is the larger of $C$ and $A$. For the Alfvén mode, propagation is restricted to the direction along the magnetic field, while for the slow mode, the dependence upon direction contains a cosine factor which goes to zero for the direction normal to the magnetic field. To develop the group velocity from this diagram we construct the locus of the normal surfaces taking into account all directions of propagation. For the Alfvén mode this degenerates to a point while for the mixed modes more complex patterns unfold. The group wave front is determined geometrically by the locus generated by the family of normals to the phase fronts, figure 5 [Sears, 1960]. Inspecting the peculiar group locus one sees directly that for the slow mode the wave normal direction takes on many values, including that perpendicular to the magnetic field. However, the velocity drops to zero in this latter instance. The results of this type of representation have been shown

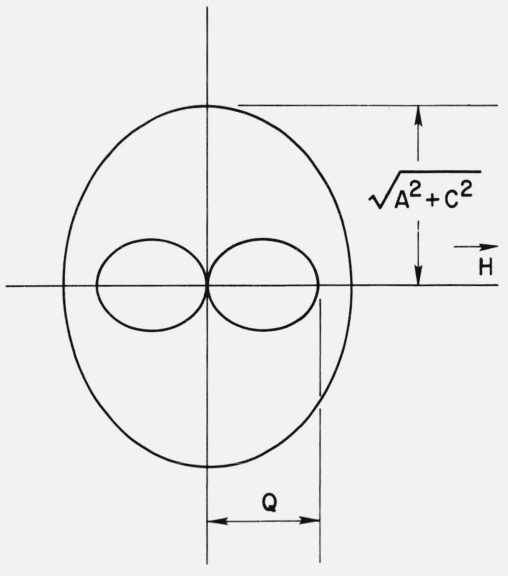

FIGURE 4. Surfaces of constant phase for the fast and slow modes. $A$ is the Alfvén velocity; $C$ the sound velocity; and $Q$ is defined as the smaller of the sound velocity and Alfvén velocity. The semiminor axis of the outer ellipse is given by the larger of the two velocities. The Alfvén velocity consists of two points on the field $(H)$ axis lying either on the semiminor axis of the outer ellipse or semimajor axis of the inner ellipse either on the semiminor axis of the outer ellipse or semimajo
depending upon the relative magnitude of the two velocities.

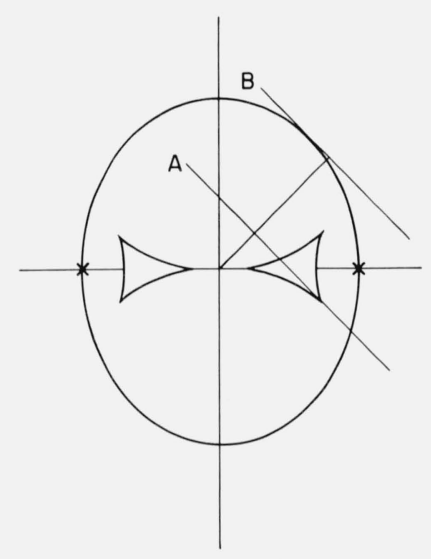

FIGURE 5. Construction of the propagation normal vectors along the surfaces of constant phase.

The locus of the normals define the surface of constant group velocity. The figure is to be rotated about the axis of the magnetic field so as to create a figure of revolution [Sears, 1960].

by Sears [1960] to be directly applicable to the construction of Mach cones, wakes, and their directions with respect to the shock. In particular, figure 6 shows several cases for the flow velocity vector parallel to the magnetic field, with the Mach cone being symmetric. To develop the Mach cone it is necessary that the flow be supersonic to the mode in question, and that it be possible to construct tangent lines from the velocity vector to the group velocity surface. In figure $6 \mathrm{~b}$ such a construction is impossible and the development of a Mach cone is forbidden. In figure 6c the flow is subsonic in the sense of the magnitude of the velocity. Yet a Mach cone can be constructed albeit in the upstream direction. It is seen immediately upon consideration that the Mach cones become asymmetric when the velocity vector is not colinear to the direction of the magnetic field. Since the Mach cone defines the boundary surface for the shock of infinitesimal strength, it follows that an MHD model 

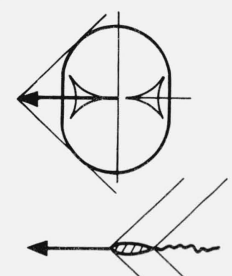

(a)

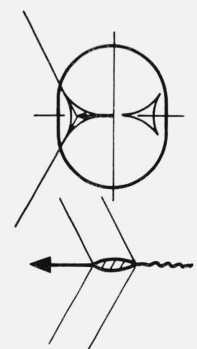

(c)

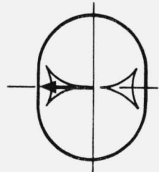

(b)

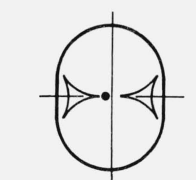

moses

(d)
FIGURE 6. Mach cones defining the locus of limiting characteristics and wakes for several cases having fluid velocity along the axis of the magnetic field but with different amplitudes [Sears, 1960].

for the bow wave flow in front of the earth must be approached with considerable caution. It is hoped that later experiments will allow an examination of the applicability of these properties of MHD waves to the case of the bow shock.

\section{Post-Shock or Downstream Flow}

Usually labeled the transition region, this volume is occupied by the shocked gas generated in the interaction process. The limited indications of theory are that collision-free shocks are not time stationary; thus the transition region may, in reality, be an extension of the initial shock with relaxation taking place over a region much thicker than the few gyroradii of the first pulse. Indeed the Mariner calculation suggests this, as a fit was obtained by using average values of gas and field parameters downstream of the shock.

The downstream flow is an especially interesting problem from the standpoint of plasma physics since the general development of turbulence and stochastic acceleration experiments in collision-free plasmas can be carried out in this region of space. This space is known to be occupied, at least sometimes, by a highly disordered field, a sample of which is shown in figure 7. Spectra of the magnetic turbulence suggest that two separate processes are present [Sonett, 1963]. The large pulses seen in figure 7 show a strong lowfrequency component while a frequency dependent component appears at higher frequencies (fig. 8). There is little similarity to either of the two basic modes of turbulence suggested by Chandrasekhar [1955], a velocity or magnetic mode depending upon the partition of energy between field and gas. That this gas is in motion and is sweeping around the magnetosphere in the usual manner of blunt-body flow is suggested by the experiment of Wolfe and Silva [1964]. Figure 9, taken from their paper, gives a view of the direction of the plasma arriving during the events of October 7, 1962, and for the period of time during those events when the satellite was within the shocked gas of the transition layer. It is observed that the flow originates from a region making an angle of about $35^{\circ}$ with respect to the sun. The details of this experiment are reported elsewhere and only the results are quoted here. Since the spacecraft was on the dawn flank of the magnetosphere, consideration of the geometry shows that the flow was from the general direction of the stagnation point and clearly not from the solar direction. We take this to be evidence of transition-layer flow of the blunt-body type.

Observation of the turbulence of the magnetic field in the transition layer is made in a number of satellite and space probe experiments, though three-dimensional cross-correlation functions needed for a definition of the exact properties cannot be made because of limitations either in experiments or in the manner of reading out the data. At the present stage of understanding together with the restricted satellite data available it becomes unfeasible to attempt to separate wave motions from stationary or pseudostationary variations in downstream plasma properties. Thus such matters as correlation tensors b-tween the pertinent plasma parameters must be regarded as quantities which are mixed with time-variant wavelike motions as well as the streaming properties of the gas.

Since the process of particle acceleration is presently the subject of conjecture, we shall not deal with it here. Also the experimental processes observed are generally the subject of a separate treatment and shall not be dealt with except for summary remarks. General conclusions from experiments prior to those of Freeman, Van Allen, and Cahill [1963] are shown in figure 10. From the Pioneer IV experiment of $\mathrm{Van}$ Allen and Frank [1959] it was shown that hot electrons
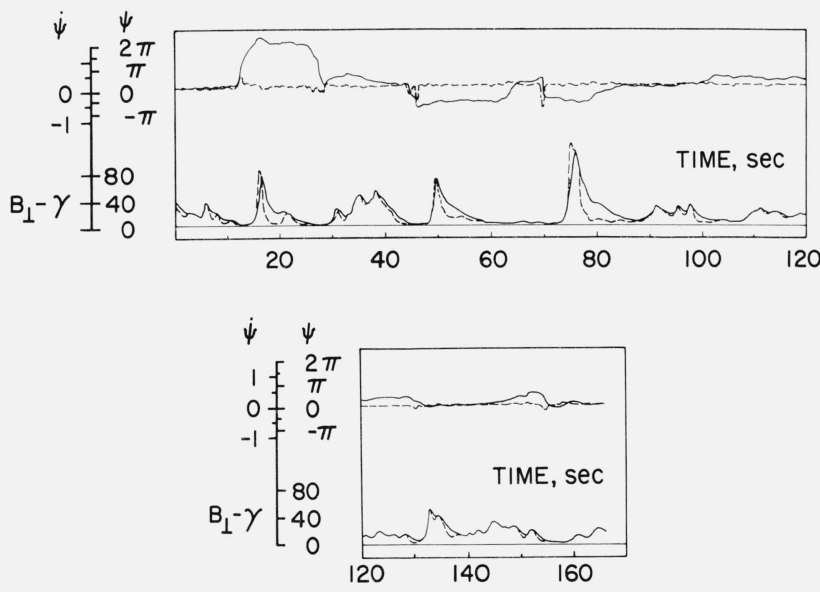

FIGURE 7. Swath of two-dimensional cut in the transition region field showing amplitude, and direction and rate of turning.

Reversals in field direction are distinctly seen and generally are associated with zeros in the magnitude [Sonett, 1963]. 


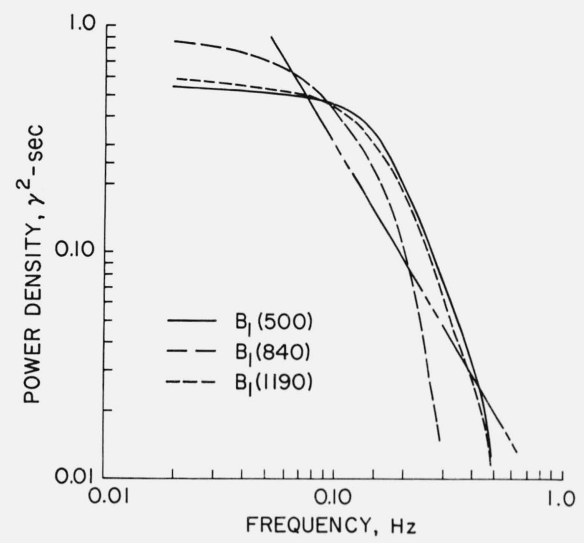

FIGURE 8. Longitudinal wave power density in the transition region magnetic field for three typical swaths of data.

The straight line is for a theoretical Kolmogoroff spectrum. Magnetometer response was about $2 \mathrm{~Hz}$ between half-power points [Sonett, 1963].

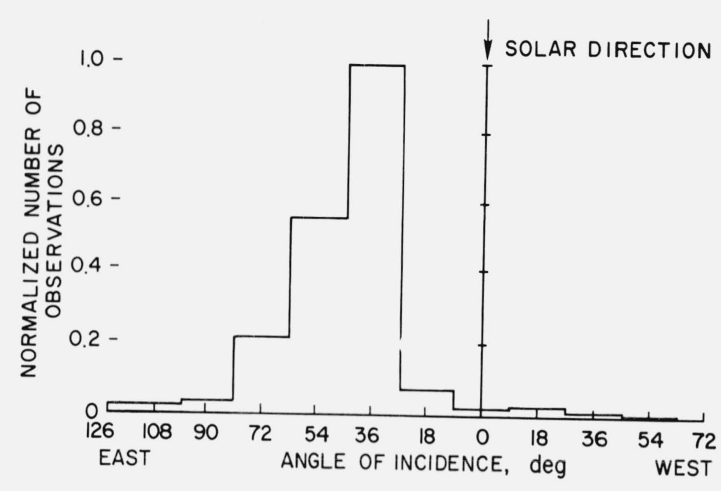

FIGURE 9. Angle of arrival of peak plasma flux versus number of observations.

The peak at about $35^{\circ}$ is approximately toward the subsolar point distinctly showing the expected blunt body flow pattern [Wolfe and Silva, 1964].

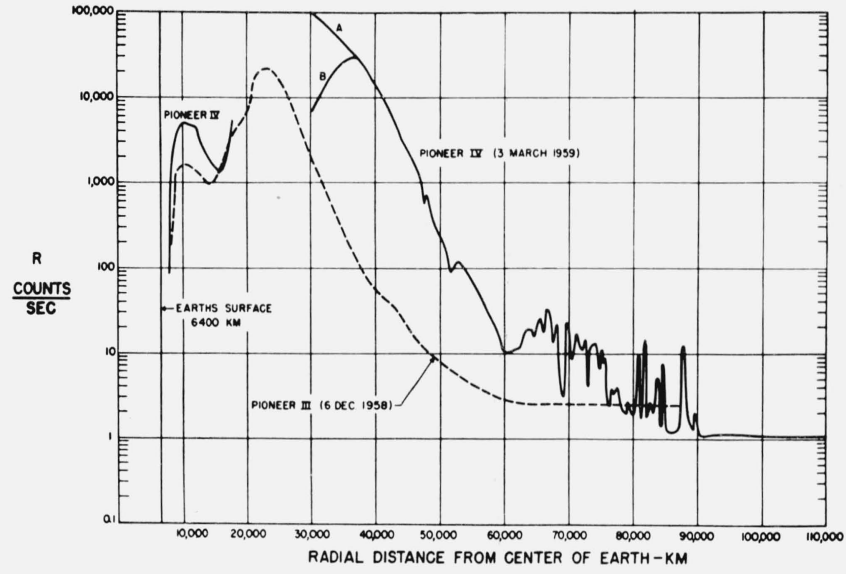

Pioneer 五 (Van Allen, 1959)
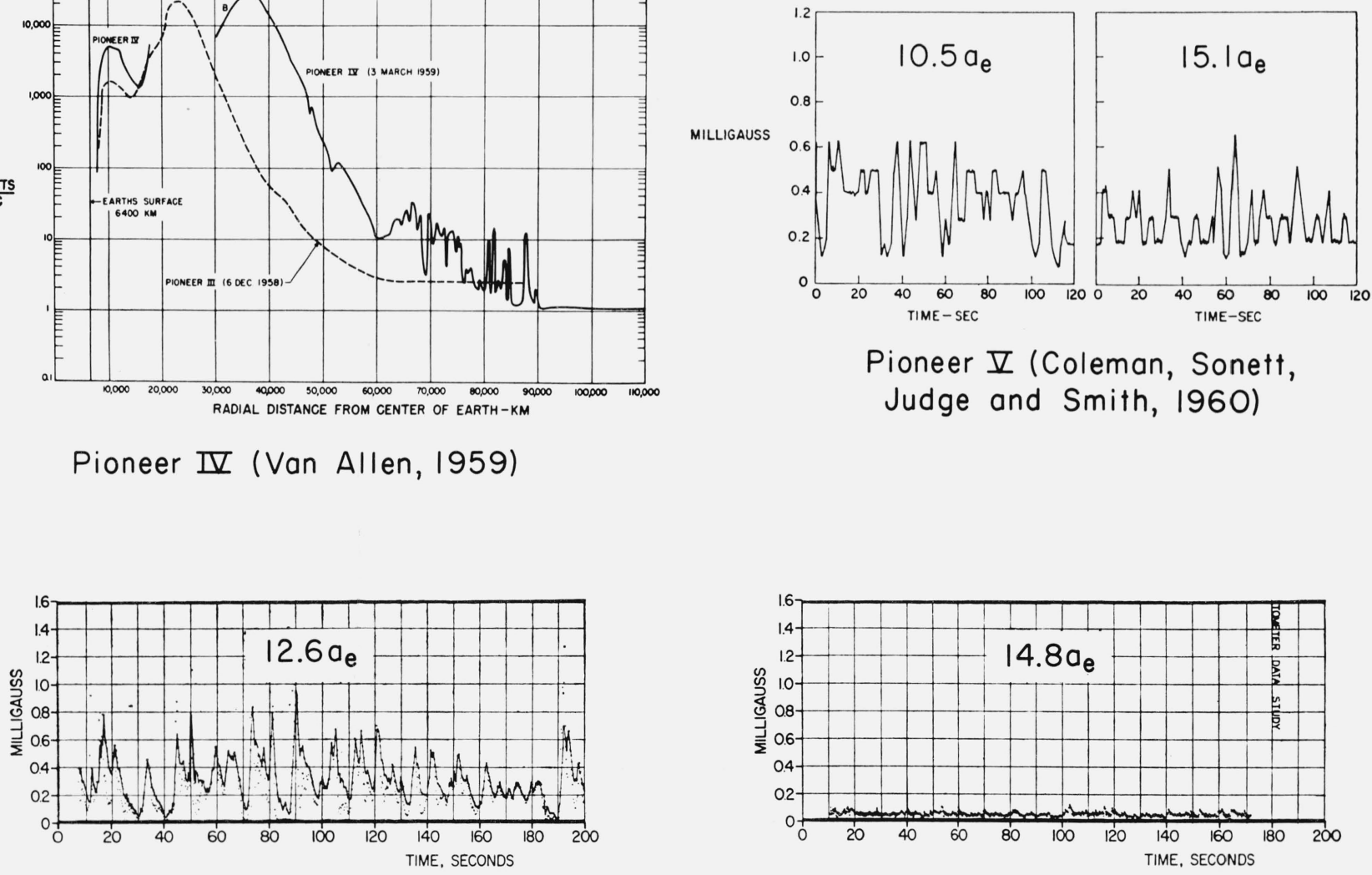

\section{Pioneer I (Sonett, Smith and Sims, 1960)}

FIGURE 10. Summary of early observations of the transition region of the magnetosphere showing Pioneer I, Pioneer IV, and Pioneer V data [Spreiter and Jones, 1963]. 
in the range of tens of kilovolts could exist in the transition zone. This result together with the early observations of the interaction region between the solar wind and the magnetosphere is shown in this figure. Reference to the discussion concerning the electron observations of Van Allen are given by Sonett [1963].

In regard to the transmission properties of the transition region insofar as propagating disturbances from the solar wind to the surface of the earth, it is well known that sudden commencements are seen on the surface ostensibly caused by rapid compression of the magnetospheric boundary in response to a sudden change in the momentum density flux of the solar wind. Thus it appears that whatever the details of the flow pattern it seems safe to regard the transition region as capable of transmitting pressure changes from the free-stream wind to the magnetosphere with the subsequent generation of magnetohydrodynamic waves which we regard as transmitted to the surface of the earth to produce the sudden commencement or impulse.

\section{Recent Experiments}

The Explorer 18 or IMP satellite is the latest in a series of spacecraft designed for investigating the magnetosphere and adjacent interplanetary space. Basically, this satellite had an orbit of extreme eccentricity with apogee at about $32 R_{e}$. The extreme orbit meant that the period was 3 to 4 days and that the spacecraft spent about two-thirds of its time beyond $20 R_{e}$. The data system is quite complex, utilizing a data burst technique where a high data rate is interspersed with blanks, for any one instrument. Only the 5-min averages of the magnetometer data have been reported to date, and these tend to confirm earlier spacecraft findings regarding the magnetic properties of the transition region and the rather turbulent character of the interplanetary gas. The data have also yielded substantial information regarding the existence of a sudden discontinuity in the magnetic field which is interpreted as a shock wave [Ness, Searce, and Seek, 1964] though the plasma experiments do not correspond to this view rigorously all the time.

Two plasma experiments were carried on this spacecraft; these were of radically different design, one being a Faraday cage and the other an electrostatic analyzer. The limitations of telemetry allowed space to be divided into three sectors for the electrostatic analyzer, henceforth referred to as the Ames experiment [Wolfe, Silva, and Myers, 1964]. Threshold for this instrument was about $10^{-14} \mathrm{~A}$, corresponding to $3 \times 10^{5}$ $\mathrm{H}^{+} / \mathrm{cm}^{2}$ sec. The energy resolution was 6.5 to 7.5 percent and the electrometer was of the peak reading variety, reading the peak current in each of the angular sectors. Results of this experiment are complex and the reader is referred to the original paper for details. The results show variations in the direction of arrival of flux changing from a highly directed beam when in the solar wind to a pseudoisotropic flux when in the transition region, though this behavior is by no means common to all the orbits. When this type of behavior was noted, the character of the velocity spectrum also underwent significant changes, confirming the view that the solar wind is a relatively cool gas compared to the gas of the transition region. Some of the preliminary results are summarized in figure 11 which shows a display of the boundary crossings according to a prescribed set of rules given by Wolfe et al., together with a comparison to the theory of Spreiter and Jones [1963]. The time the plasma probe crossed a zone was indicated by the appearance or disappearance of the characteristic spectrum in both velocity and angular distribution. The crossing of the inner boundary, i.e., the termination of the regular magnetosphere, was indicated by the cessation of sensible readings on the plasma probe for inbound orbits and an onset for outbound cases. The dashed lines are those cases where the definition was difficult to satisfy. In particular, there were many times when an outer or shock boundary was difficult or impossible to find and at other times the time of crossing was different from that indicated by the magnetometer. At other times the agreement was quite good and the supposition for the shock appears to rest upon reasonable data. This may suggest a high degree of variability in the gas parameters or perhaps tends to underline present ignorance of the true meaning of these experiments. Results of some of the other experiments of this spacecraft tend to confirm the view that the transition region contains a remarkably hot gas. Little can be said regarding the manner of leakage of this gas into the regular magnetosphere and whether it supplies some or all of the flux of electrons of the Van Allen radiation.

Calculations being carried out by Olbert (private communication) seem to confirm that collision-free shocks require a scale far in excess of the gyroradius geometric mean to damp into the post-shock downstream state irreversibly. These calculations, carried out for the bow shock and based upon the plasma experiment of Bridge et al., [1964] and Ness et al., [1964] also suggest the applicability of the MHD Rankine-Hugoniot equations. In the instance of Olbert's calculation it is found that the downstream

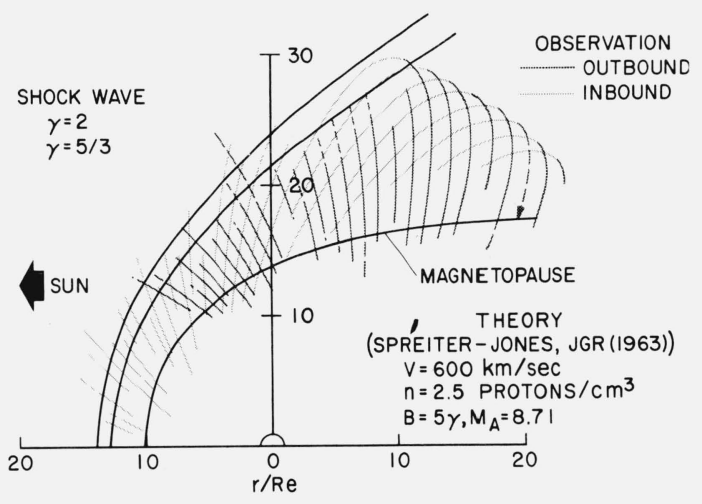

FIGURE 11. Locus of outward and inward bound IMP orbits as the satellite crossed the transition region.

The definition of crossing for the purposes of this diagram is based upon the Wolfe experiment and will vary from the findings of other experimenters because of the differing instrument response and definition of crossing. The reference idealized shocks for the two different ratios of specific heat are from the calculations of Spreiter and Jones [1963]. different ratios of specific heat are from the calculat
The $\gamma=2$ case is the outermost of the two shocks. 
gas has a very low ratio of specific heats, this conclusion being arrived at from the very large density jump across the shock. Insofar as the generation of entropy is regarded as the true test for a shock even in the collision-free domain, it appears that the properties of the downstream flow directly behind the onset of the shock will play a leading role in the next few years in establishing a foundation for collision-free shock theory.

The very preliminary status of reporting of the data from the majority of the IMP experiments together with the necessary brevity of this report preclude the possibility of reviewing the majority of the results. It is probable that forthcoming results from the IMP series will provide much of the information required in order to obtain a meaningful physical basis for much of what today must be regarded as only partially understood physical phenomena.

The author acknowledges several stimulating discussions with J. R. Spreiter and, in particular, his calling attention to the work of Sears regarding shock modes.

(Paper 69D8-537)

\section{References}

Axford, W. I., and C. O. Hines (1961), A unifying theory of highlatitude geophysical phenomena and geomagnetic storms, Can. J. Phys. 39, No. 10, 1433-1464.

Beyer, M. (1953), Brightness of comets and solar activity, Soc. Roy. Sci. Liége 13, No. 1-2, 276-86.

Bright, H., A. Egidi, A. Lazarus, E. Lyons, and L. Jacobson (May 1964), Preliminary results of plasma measurements on IMP-A COSPAR, Preprint, Florence, Italy.
Chandrasekhar, S. (Dec. 1955), Hydromagnetic turbulence II. An elementary theory, Proc. Roy. Soc. London, A233, No. 1194, 330-50.

Davis, L., Jr., E. J. Smith, P. J. Coleman, Jr., and C. P. Sonett (1964), Interplanetary magnetic measurements, presented at Solar Wind Conference, JPL, Pasadena, Calif., April 1-4 1964.

de-Hoffmann, F., and E. Teller (1950), Magneto-hydrodynamic shocks, Phys. Rev. 80, No. 4, 692-703.

Freeman, J. W., J. A. Van Allen, and L. J. Cahill (1963), Explorer 12 observations of the magnetosphere boundary and the associated solar plasma on September 13, 1961, J. Geophys. Res. 68, No. 8, 2121-2130.

Greenstadt, E. W. (April 1963), Effect of solar activity regions on the interplanetary magnetic field, Astrophys. J. 137, No. 3, 999-1002.

Ness, N. F., C. S. Scearce, and J. B. Seek (1964), Initial results of the IMP-1 magnetic field experiment, J. Geophys. Res.69, No. 17, 3531-3569.

Parker, E. N. (Nov. 1958), Dynamics of the interplanetary gas and magnetic fields, Astrophys. J. 128, No. 3, 664-676.

Sears, W. R. (Oct. 1960), Some remarks about flow past bodies, Rev. Mod. Phys. 32, No. 4, 701-705.

Sonett, C. P. (1963), The distant geomagnetic field 4: Microstructure of a disordered hydromagnetic medium in the collisionless limit, J. Geophys. Res. 68, No. 5, 1265-1294.

Sonett, C. P., D. S. Colburn, L. Davis, Jr., E. J. Smith, and P. J. Coleman, Jr. (1964), Evidence for a collision-free magnetohy. drodynamic shock in interplanetary space, Phys. Rev. Letters 13, No. 5, 153-156.

Spreiter, J. R., and W. P. Jones (1963), On the effect of a weak interplanetary magnetic field on the interaction between the solar wind and the geomagnetic field, J. Geophys. Res. 68, No. 12, $3555-3564$.

Spreiter, J. R., and A. L. Summers (1963), Effect of uniform external field and oblique incidence of the solar wind on the terminal shape of the geomannetic field, NASA TR R-181.

Snyder, C. W., and M. Neugebauer (1964), Interplanetary solar wind measurements by Mariner II, Space Res. IV.

Van Allen, J. A., and L. A. Frank (1959), Radiation measurements to $658,300 \mathrm{~km}$ with Pioneer IV, Nature 184, No. 4682-1, 219-24.

Walters, G. K. (1964), Effect of oblique interplanetary magnetic field on shape and behavior of the magnetosphere, J. Geophys. Res. 69, No. 9, 1769-83.

Wolfe, J. H., and R. Silva (1965), Explorer 14 plasma probe observations during the October 7, 1962, geomagnetic disturbance, J. Geophys. Res., l August 1965 (in press).

Wolfe, J. H., R. Silva, and M. Myers, Observations of the solar wind during the flight of IMP-1 (in preparation - to be submitted to J. Geophys. Res.). 